\title{
Bioelectric Model of Atrial Fibrillation: Applicability of Blind Source Separation Techniques for Atrial Activity Estimation in Atrial Fibrillation Episodes
}

\author{
JJ Rieta, F Castells, C Sánchez, D Moratal-Pérez, J Millet \\ Bioengineering Electronic and Telemedicine Research Group \\ Polytechnic University of Valencia, Spain
}

\begin{abstract}
In this contribution, we present the theoretical justification that give support to the suitability of Blind Signal Separation (BSS) techniques for the estimation of the atrial activity $(A A)$ present in ECGs of persistent atrial fibrillation $(A F)$. The application of BSS methods to this problem needs the fulfillment of several conditions regarding $A A$, ventricular activity (VA) and the fashion in which both activities arise on the body surface, that will be justified along the paper. To empirically validate the model, an ICA method is applied to 10 real 12-Lead recordings of AF. The identification of $A A$ is put forward based on kurtosis and spectral analysis. The kurtosis value of the estimated AA was always below zero $\left(k_{A A}=-0.36 \pm 0.14\right)$, while for the VA was above $20\left(k_{V A}=30.44 \pm 7.83\right)$. As conclusion, the verification of the AF bioelectric model makes feasible the application of BSS, and this contribution has indeed justified, for the first time, the theoretical background that supports the applicability of these techniques and empirically its usefulness to solve the AA extraction problem.
\end{abstract}

\section{Introduction}

Atrial fibrillation (AF) is the most common sustained arrhythmia encountered by clinicians and occurs in approximately $0,4 \%$ to $1,0 \%$ of the general population. Its prevalence increases with age, and up to $10 \%$ of the population older than 80 years has been diagnosed with AF. With the projected growth of the elderly population, the prevalence of AF will certainly increase [1]. There is also increasing awareness that atrial fibrillation is a major cause of embolic events which in $75 \%$ of cases are complicated by cerebrovascular accidents [2]. For the study of AF it is necessary to separate AA from VA in the ECG. This can be carried out by direct cancellation of VA [3], but also the use of BSS techniques has demonstrated to be very effective for the AA extraction problem y AF $[4,5]$. Nevertheless, the applicability of BSS for the separation of AA and AV has not been formally justified. Hence, the goal of this work is to justify the fulfillment of the conditions for AA and AV that allow its separation via BSS from the ECG in AF.

\section{BSS approach to atrial fibrillation}

If BSS methods are to be applied to the AA extraction from the 12-lead ECG in AF episodes, the fulfillment of several basic considerations regarding AA, VA and the fashion in which both activities arise on the body surface must first be justified: independence of the sources, nongaussianity, and nonorthogonal observations generated by instantaneous linear mixing of the bioelectric sources [6]. This section begins with an outline of the basic mathematical principles behind the BSS of instantaneous linear mixtures. Then, physical mechanisms of AF generation will give support to the independence and nongaussianity of AA and VA. Next, the validity of the instantaneous linear mixing model for the ECG is endorsed through the matrix solution for the forward problem of electrocardiography. The corroboration of these conditions make it possible to assume that the ECG of a patient in AF satisfies the BSS instantaneous linear mixture model, thus justifying the application of BSS techniques.

\subsection{BSS principles}

The BSS consists in recovering a set of source signals from the observation of linear mixtures of the sources. The term "blind" emphasizes that nothing is known about the source signals or the mixing structure, the only hypothesis being the source mutual independence [6]. Mathematically, let us denote $\mathbf{s}(t) \in \Re^{N}$ the vector that represents the $N$ source signals, and $\mathbf{x}(t) \in \Re^{M}$ the $M$ sensor output vector, i.e. the observation vector, where it is assumed that $M \geq$ $N$, so that there are at least as many sensors as sources. In the noiseless case, the BSS model for instantaneous linear mixtures reads

$$
\mathbf{x}(t)=\mathbf{A} \mathbf{s}(t)
$$

where $\mathbf{A} \in \Re^{M \times N}$ is the unknown mixing matrix. The objective of BSS is to estimate $\mathbf{s}(t)$ and $\mathbf{A}$ from the exclusive knowledge of $\mathbf{x}(t)$. A comprehensive reading of BSS principles can be found in [7] 


\subsection{Mechanisms of atrial fibrillation}

$\mathrm{AF}$ is characterized by uncoordinated atrial activation and is due to the unpredictable conduction of these disordered impulses across the atrial myocardium [1]. Theories of the AF mechanism involve two processes [1]: enhanced automaticity in one or several foci and reentry involving one or more circuits. The focal origin of AF is supported by experimental models and appears to be more important in paroxysmal AF [2]. Nevertheless, the most widely accepted theory of persistent AF is based on the continuous propagation of multiple wavelets wandering throughout the atria $[1,2]$. The fractionation of the wave fronts as they propagate results in self-perpetuating independent wavelets. The number of simultaneous wavelets depends on the refractory period, mass and conduction velocity along the atria, and these parameters present severe inhomogeneities in AF [1]. Moreover, the self-perpetuating propensity of $\mathrm{AF}$ is justified by the electrophysiological remodelling, a phenomenon consisting in the progressive shortening of effective refractory periods, thus increasing the number of simultaneous wavelets and, as a consequence, the episode duration [1].

\subsection{Independence and nongaussianity}

During an AF episode several independent wave fronts propagate simultaneously throughout the atria but only a reduced part of them will reach the AV node. Moreover, the properties of the AV node tend to limit strongly the ventricular activation. First, the excitability of cells within the $\mathrm{AV}$ node is significantly lower than the atrial myocardium [1]. Second, the AV node demonstrates decremental conduction, where impulses may traverse only a portion of the $\mathrm{AV}$ node before blocking [2]. One clinical manifestation of this property is the concealed conduction, in which an atrial impulse, that itself does not conduct to the ventricles, may impair conduction of subsequent impulses, blocking the propagation of other impulses that otherwise would have conducted [2]. As a consequence of the aforementioned $\mathrm{AV}$ node properties, most of the atrial wave fronts do not reach conduction and are not able to produce ventricular depolarization.

On the other hand, the physical origin of the atrial wave front that produce ventricular depolarization could be very variable. This uncoordinated operation of AA and VA during an $\mathrm{AF}$ episode makes it reasonable to regard both activities as physically independent and, in turn, as generated by statistically independent sources of cardioelectric activity. The validity of the atrio-ventricular statistical-independence assumption is in line with the findings reported by other authors in the field [1, 2, 3]. With respect to nongaussianity, VA presents high values within the heart beat (QRS complex) and low values in the rest of the cardiac cycle. Hence, the histogram analysis of VA reveals an impulsive, i.e., supergaussian, behavior [8] with typical kurtosis values above 15 . On the other hand, the AA of an AF episode has been accurately modeled as a sawtooth signal consisting of a sinusoid with several harmonics [3], which behaves, statistically speaking, as a subgaussian random process. Moreover, when a QRS complex and $\mathrm{T}$ wave cancellation algorithm, like those described in $[3,8]$, is employed to cancel out VA over one ECG lead, it can be observed that the remaining ECG, mainly the AA, present a subgaussian behavior with negative kurtosis values. The nongaussian assumption of AA and VA is hence justified and will also be shown in the Results section.

\subsection{ECG instantaneous linear model}

There are several physical models to represent both the cardiac current sources and the enclosing torso shape and conductivity [9]. The combination of torso and source models to calculate the body surface potentials is known as the forward problem [10]. One solution for the forward problem relays on the calculation of the body surface potentials from the epicardial surface potentials [10]

$$
\begin{aligned}
& \mathbf{P}_{B B} \boldsymbol{\Phi}_{B}+\mathbf{P}_{B H} \boldsymbol{\Phi}_{H}+\mathbf{G}_{B H} \boldsymbol{\Gamma}_{H}=0 \\
& \mathbf{P}_{H B} \boldsymbol{\Phi}_{B}+\mathbf{P}_{H H} \boldsymbol{\Phi}_{H}+\mathbf{G}_{H H} \boldsymbol{\Gamma}_{H}=0
\end{aligned}
$$

where $\boldsymbol{\Phi}_{B}$ and $\boldsymbol{\Phi}_{H}$ are column vectors of potentials, $\boldsymbol{\Gamma}_{H}$ is a column vector of epicardial potential gradients, and the various $\mathbf{P}$ and $\mathbf{G}$ coefficient matrices are determined solely by integrations involving the geometry of the epicardial $\left(S_{H}\right)$ and body $\left(S_{B}\right)$ surfaces. Solving Eq. (3) for the matrix of epicardial potential gradients $\boldsymbol{\Gamma}_{H}$ and substituting the result into Eq. (2) yields

$$
\boldsymbol{\Phi}_{\mathbf{B}}=\mathbf{Z}_{B H} \boldsymbol{\Phi}_{H}
$$

where $\mathbf{Z}_{B H}$ is obtained by operating with the different $\mathbf{P}$ and $\mathbf{G}$ matrices. Eq. (4) define the solution to the forward problem. The elements of matrix $\mathbf{Z}_{B H}$ are transfer coefficients relating the potential at a particular point on $S_{H}$ to that at a particular point on $S_{B}$, and they depend solely on the geometry of the epicardial and body surfaces and the conductivity of the torso [10]. Obviously, Eq. (4) corresponds to a linear mixing model where a set of observations are obtained by linearly combining a set of sources. Because the mathematical operations that define the voltages for the 12-lead ECG, are only linear combinations of the body surface potentials, this do not affect at all to the aforementioned instantaneous linear mixture model of Eq. (4) for the 12-lead ECG.

This BSS model can be solved using ICA, which does not introduce any restriction on the geometrical structure of the mixing matrix (apart from the linear independence 
of its columns) and, in addition, takes into account the nongaussian nature of the source signals [11]. Observe that the application of BSS-based methods on the standard ECG can be completely justified and remarked with the duality between Eq. (1) and Eq. (4). As a consequence, the most important requirements to apply the ICA-based BSS technique, namely, source independence, nongaussianity and instantaneous non-orthogonal linear mixtures do indeed hold for the 12-lead ECG recordings of a patient with AF.

\section{Database and methods}

All signals were sampled at $1 \mathrm{kHz}$ and were preprocessed using a notch filter to cancel out mains interference ( $f_{n}=50 \mathrm{~Hz}$ ), and a band-pass filter with cut-off frequencies of 0.5 and $65 \mathrm{~Hz}$ to remove baseline wandering and reduce thermal noise. The signal database was comprised of recordings from 10 patients in AF, all of them 12-lead and 8 seconds in length.

Given the duality between the presented BSS and ECG models, the skin-electrode signal vector of one AF recording can be identified with $\mathbf{x}(t)$ in Eq. (1), where vector $\mathbf{s}(t)$ will be composed of the independent components of atrial and ventricular cardiac activities and other nuisance signals [4]. Each entry of the mixing matrix $\mathbf{A}$ will depend on the body geometry, tissue conductivity, electrode position, etc. Hence, the AA contribution to each ECG lead can be extracted, to obtain a unified AA signal, using and ICAbased method. In the present work the FastICA algorithm [7] was used due to its high performance.

The subgaussian model of AA in front of the supergaussian behavior of VA allows the identification of AA using a kurtosis-based reordering of the ICA separated sources. This will place in one side the subgaussian sources and in the other side the supergaussian sources.

To improve the AA identification after the kurtosis reordering step, the power spectral density (PSD) was computed for all the separated sources with subgaussian kurtosis $(k<0)$. The procedure consisted of obtaining the modified periodogram from the separated sources using the Welch-WOSA method with a Hamming window of 4096 points length, a 50\% overlapping between adjacent windowed sections and a 8192 points length FFT. Later, the spectral content above $20 \mathrm{~Hz}$ has been discarded due its low contribution. This way, it is possible to compare the spectral content of the AA sources with the accepted spectral content of AF $[8,5]$.

\section{Results and discussion}

After the application of the ICA method it was always possible to estimate the AA and VA contained into the episode. Fig 1 shows leads II, III, aVF and V1 from one of

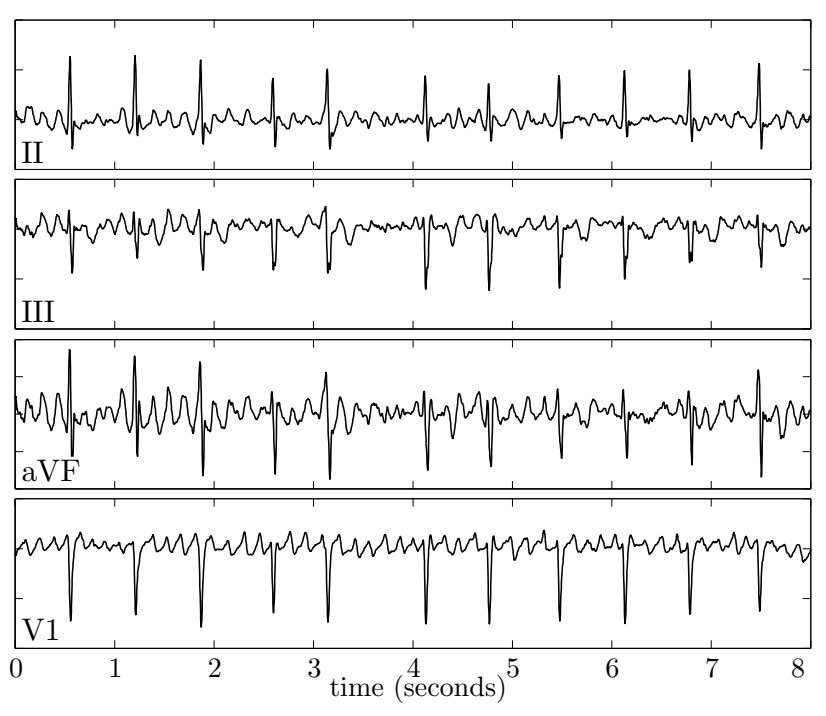

Figure 1. Leads II, III, aVF and V1 from a patient with AF.

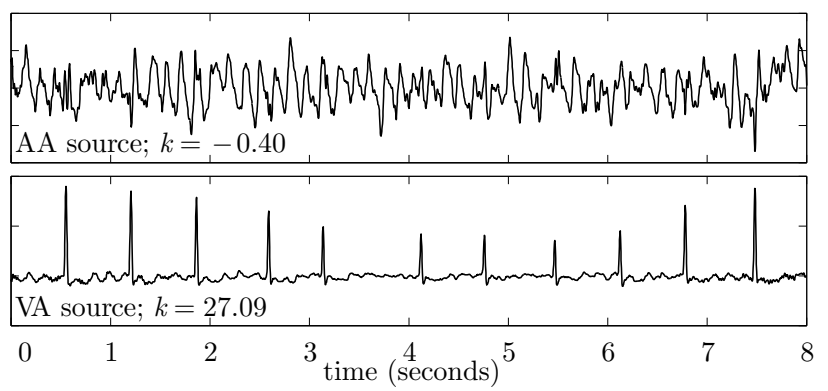

Figure 2. At the top, estimated AA source with its kurtosis value. Below, is estimated VA source for the same episode.

the patients (it is accepted that these leads have the largest AA content [1]). The result of applying ICA to this AF episode and reordering the estimated sources as a function of its kurtosis gives, in one extreme of the set of separated sources, the estimated AA and, in the other extreme, the VA. Fig. 2 plots these extremes.

To validate the statistical behavior of AA and VA, Fig. 3 shows the histograms of the signals from Fig. 2 identified as AA and VA along with a superimposed normal distribution for comparison. As can be seen, the AA present a subgaussian distribution whereas the VA is clearly supergaussian. Table 1 shows the results for the set of 10 patients analyzed. As can be observed, the kurtosis for the estimated AA was always below zero and, for the VA case, it was clearly above zero, thus corroborating the previously justified statistical behavior of both cardiac activities.

In general, the subgaussian behavior of the estimated AA is not so far from Gaussianity. Nevertheless, this result has not to be considered as a problem for the separation of AA from Gaussian noise because the power of noise in the ECG is much more smaller that AA, as has been proved 

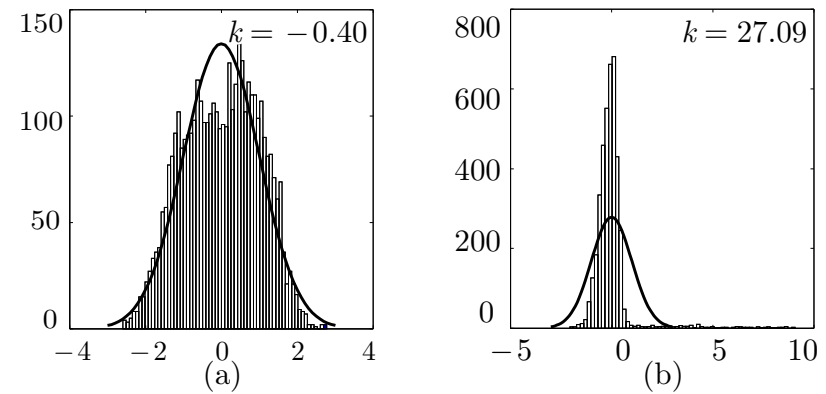

Figure 3. Histograms of the estimated atrial and ventricular sources obtained from Fig. 2. a) Atrial activity histogram. b) Ventricular Activity histogram.

\begin{tabular}{|c|c|c|c|c|c|}
\hline & Pat.01 & Pat.02 & Pat.03 & Pat.04 & Pat.05 \\
\hline$k_{A A}$ & -0.40 & -0.55 & -0.11 & -0.38 & -0.36 \\
\hline \multirow[t]{2}{*}{$k_{V A}$} & 27.09 & 28.26 & 36.67 & 48.61 & 28.93 \\
\hline & Pat.06 & Pat.07 & Pat.08 & Pat.09 & Pat.10 \\
\hline$k_{A A}$ & -0.31 & -0.51 & -0.41 & -0.12 & -0.40 \\
\hline$k_{V A}$ & 26.65 & 25.75 & 20.22 & 34.44 & 27.71 \\
\hline$\overline{k_{A A}}$ & -0.3 & \pm 0.14 & $\overline{k_{V A}}$ & $=30.4$ & $=7.83$ \\
\hline
\end{tabular}

Table 1. Kurtosis values for the patients in the database (Pat.01 to 10) of the estimated AA $\left(k_{A A}\right)$ and VA $\left(k_{V A}\right)$. The mean value and standard deviation, in each case, for all the patients analyzed are $\overline{k_{A A}}$ and $\overline{k_{V A}}$ respectively.

in the results. Moreover, it could be possible to separate the AA from Gaussian noise via their very dissimilar time and frequency behavior, as proved in [12].

\section{Conclusions}

The present contribution has proven the suitability of the ICA-based methods to separate AA and VA in patients with AF due to the justification of the sequel facts: firstly, in atrial arrhythmia episodes the bioelectric sources of the heart generating AA and VA can be regarded as uncoupled and statistically independent. Secondly, both activities present a non-Gaussian behavior and, finally, AA and VA are manifested at the body surface as an instantaneous linear mixture in which the mixture depends on the electrode position of the ECG. These considerations make feasible the application of ICA to solve the AA extraction problem in $\mathrm{AF}$ and this contribution has justified theoretically the applicability of these techniques. Later, the theoretical bioelectric BSS model of AF has been validated empirically via the application of ICA to real ECG recordings.

\section{Acknowledgements}

The authors would like to thank cardiologists R. Ruiz Granell, S. Morell, F. J. Chorro and R. García Civera, from the Universitary Clinical Hospital of Valencia, for their clinical advice and kind help in obtaining the signals. This work was partly funded by the research incentive program of the UPV, CTIDIA/202/053 and TIC2002-00957.

\section{References}

[1] Fuster V, Ryden LE, Asinger RW, et al. ACC/AHA/ESC guidelines for the management of patients with atrial fibrillation. Journal of the American College of Cardiology 2001; 38(4):1266i-1266lxx.

[2] Falk RH. Medical progress: Atrial fibrillation. New England Journal of Medicine 2001;344(14):1067-1078.

[3] Stridh M, Sörnmo L. Spatiotemporal QRST cancellation techniques for analysis of atrial fibrillation. IEEE Trans Biomed Eng 2001;48(1):105-111.

[4] Rieta JJ, Zarzoso V, Millet J, Garcia R, Ruiz R. Atrial activity extraction based on blind source separation as an alternative to QRST cancellation for atrial fibrillation analysis. IEEE Computers in Cardiology 2000;27:69-72, Boston, MA, USA.

[5] Langley P, Bourke JP, Murray A. Frequency analysis of atrial fibrillation. IEEE Computers in Cardiology 2000;27:65-68.

[6] Cardoso JF. Blind signal separation: Statistical principles. Proceedings of the IEEE 1998;86(10):2009-2025.

[7] Hyvarinen A, Karhunen J, Oja E. Independent Component Analysis. John Wiley \& Sons, Inc., 2001.

[8] Shkurovich S, Sahakian AV, Swiryn S. Detection of atrial activity from high-voltage leads of implantable ventricular defibrillators using a cancellation technique. IEEE Trans Biomed Eng 1998;45(2):229-234.

[9] Malmivuo J, Plonsey R. Bioelectromagnetism: Principles and Applications of Bioelectric and Biomagnetic Fields. Oxford University Press, 1995.

[10] Gulrajani RM. The forward and inverse problems of electrocardiography. IEEE Engineering in Medicine and Biology Magazine 1998;17(5):84-101.

[11] Rieta JJ, Castells F, Sanchez C, Igual J. ICA applied to atrial fibrillation analysis. International Conference on Independent Component Analysis and Blind Signal Separation ICA 2003;4:59-64, Nara, Japan.

[12] Castells F, Ruiz R, Rieta JJ, Millet J. An integral atrial wave identification approach based on spatiotemporal source separation: Clinical validation. IEEE Computers in Cardiology 2003;30:In press. Thessaloniki, Greece.

Address for correspondence:

José Joaquín Rieta

EPSG-UPV

Carretera Nazaret-Oliva s/n 46730, GANDIA (Valencia), Spain

E-mail: jjrietadeln.upv.es 